\title{
Correlation between glioblastoma stem-like cells and tumor vascularization
}

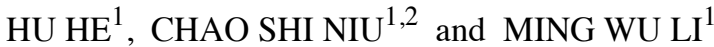 \\ ${ }^{1}$ Department of Neurosurgery, Anhui Provincial Hospital Affiliated to Anhui Medical University; \\ ${ }^{2}$ Molecular Neurobiology and Neural Regeneration and Repairing Laboratory, Anhui Provincial \\ Stereotactic Neurosurgical Institute, Hefei, Anhui Province 230001, P.R. China
}

Received August 10, 2011; Accepted September 14, 2011

DOI: $10.3892 /$ or.2011.1484

\begin{abstract}
Glioblastoma multiforme (GBM) is the most lethal type of brain tumor. The formation of abnormal, dysfunctional tumor vasculature and glioblastoma stem-like cells (GSCs) are believed to be the major components of the inability to treat these tumors effectively. We analyzed 70 glioblastoma samples by immunohistochemistry and double immunofluorescence staining. The immunohistochemical expression of the putative brain tumor stem cell markers CD133 and Nestin in paraffin sections was analyzed using morphometry. In all GBM samples, CD133 or Nestin was expressed in tumor and endothelial cells. Double immunofluorescence stainings showed that the two different marked GSCs were found accumulated around the $\mathrm{CD} 31^{+}$blood vessels and CD133/CD31 or Nestin/CD31 co-expression was found in the endothelial cells and GSCs. Furthermore, the vascular endothelial growth factor (VEGF) and the endothelial marker CD31 were co-expressed in GSCs. Therefore, GSCs not only showed distinct perivascular distribution but were capable of differentiating into endothelial cells. We demonstrate that GSCs contribute directly to the tumor vasculature by endothelial cell differentiation. GSCs and tumor vascularization are closely related to each other, not only in the regional distribution but also in biological function. These findings describe a new mechanism for tumor vasculogenesis and may provide new insights for targeted therapy against brain tumors.
\end{abstract}

\section{Introduction}

Treatment of adult brain tumors, in particular glioblastoma, remains a significant clinical challenge, despite modest advances in surgical technique, radiation and chemotherapeutics. Standard-of-care includes maximal surgical resection,

Correspondence to: Dr Chao Shi Niu, Department of Neurosurgery, Anhui Provincial Hospital Affiliated to Anhui Medical University, 17 Lujiang Road, Hefei, Anhui Province 230001, P.R. China E-mail: niuchaoshi@126.com

Key words: glioblastoma, glioblastoma stem-like cells, CD133, Nestin, vasculogenesis chemoradiotherapy, and adjuvant chemotherapy with median overall survival of 12-15 months (1). Growing evidence suggests that GSCs may be generated from neural stem cells (NSCs) as they share many similar properties. Nestin and CD133 are regarded as markers of both NSCs and GSCs, and are used to identify GSCs (2). Calabrese et al (3) showed that endothelial cells interact selectively with $\mathrm{Nestin}^{+} / \mathrm{CD} 133^{+}$brain cancer cells in culture and supply secreted factors that maintain these cells in a self-renewing and undifferentiated state. Increasing the number of endothelial cells or blood vessels in orthotopic brain tumor xenografts expanded the numbers of self-renewing $\mathrm{Nestin}^{+} / \mathrm{CD} 133^{+}$cancer cells and accelerated the initiation and growth of tumors. Another in vitro study (4) showed that CD133+ glioblastoma cells consistently secreted markedly elevated levels of VEGF suggesting a close link between $\mathrm{CD} 133^{+}$tumor cells and angiogenesis. Therefore, brain capillaries play a key role in leading brain tumor stem cells (BTSCs) to uncontrolled proliferation and tumorigenesis.

The process of angiogenesis is essential for tumor growth and development of metastasis (5). Traditionally, the tumor vasculature has been considered to be derived from pre-existing blood vessels through a process of angiogenesis $(6,7)$. However, increasing data suggest that the tumor vasculature may be derived from cancer cells through a process of vasculogenesis $(8,9)$. Bruno et al $(10)$ identified a $\mathrm{CD} 133^{+}$renal progenitor cell population in renal carcinomas that may differentiate into endothelial cells and favor vascularization and tumor growth. Hence, the tumor-derived blood vessels may be more important than pre-existing blood vessels for glioma growth.

The objective of this study was to assess the expressions of the stem cell markers Nestin and CD133 in glioblastoma samples by immunohistochemistry in order to determine whether the $\mathrm{CD}_{133}{ }^{+}$or the Nestin ${ }^{+}$cells accumulated around the brain capillaries. Moreover, double immunofluorescence staining of CD133/Nestin, CD133/CD31, Nestin/CD31 and VEGF/CD31 were performed to evaluate the correlation between glioblastoma stem-like cells and tumor vascularization.

\section{Materials and methods}

Patients and preparation of tissues. Tumor tissues from patients underwent initial surgery of glioblastoma (70 patients) between July 11, 2007 and February 7, 2010 at Anhui Provincial 
Hospital affiliated to Anhui Medical University were included in this study. The patients had received no prior treatment to the craniotomy. The age of the patients varied from 6-79 years and the average age was 64.7. In addition, the male-female ratio of patients was 1.6:1.

Fresh glioblastoma samples from all patients were fixed in $4 \%$ neutral-buffered formalin and paraffin-embedded. Threemicrometer sections were cut on a microtome and stained routinely with hematoxylin and eosin to define representative tumor regions. All glioblastoma samples were classified according to the World Health Organization guidelines 2007.

Immunohistochemistry. For immunohistochemical studies, endogenous peroxidase was neutralized with $3 \% \mathrm{H}_{2} \mathrm{O}_{2}$ in methanol (10 $\mathrm{min})$ after antigen retrieval in $0.1 \mathrm{M}$ citrate buffer (pH 5.8) at $95^{\circ} \mathrm{C}$ for $5 \mathrm{~min}$ and cooled at $25^{\circ} \mathrm{C}$ for $1 \mathrm{~h}$. Sections were blocked with normal goat serum $(10 \mathrm{~min})$, then treated with the following primary antibodies overnight at $4^{\circ} \mathrm{C}$; rabbit antiNestin polyclonal antibody (1:200; Abcam, Cambridge, UK) or mouse anti-CD133 monoclonal antibody (1:40; Miltenyi Biotec, Bergisch Gladbach, Germany). Negative control sections were incubated with PBS instead of the primary antibody. After treatment with biotinylated secondary antibody, color reactions were performed with diaminobenzidine (DAB) (Maxim, Fuzhou, P.R. China), and counterstained with Mayer's hematoxylin. Sections were dehydrated in an ascending alcohol series, cleared in Histoclear (Fisher Scientific, Waltham, MA, USA) and coverslipped with DPX (Merck, Darmstadt, Germany). Immunohistochemical staining for CD133 or Nestin was scored under a microscope. The number of positive blood vessels and cells in ten representative microscopic fields were counted and the percentages were calculated.

Double immunofluorescence staining. For double immunofluorescence staining, the same protocol described above was used. After detection of CD133 using the Catalyzed Signal Amplification II kit with fluorescence amplification (FITC) (1:100; Beijing Zhongshan Golden Bridge Biotechnology Ltd., Beijing, P.R. China), the sections were incubated with antibodies against either the intermediate filament protein marker Nestin (1:200; Zymed, San Diego, CA, USA) or the endothelial marker CD31 (1:100; Maxim) followed by Cy3-labeled secondary antibody (1:100; Maxim). Nuclei were counterstained with 4',6-diamidino-2-phenylindole (DAPI) (1:1,000; Maxim). The same method was applied to detect VEGF (1:100; Maxim)/ CD31, Nestin/CD31. Adjacent sections were stained with single primary antibody in order to validate the double staining. Fluorescent images were captured with an Olympus BX51 microscope using DAPI, FITC and Cy3 filters.

Culture of glioblastoma stem-like cells. A few minutes after tumor removal, tissues were minced with scissors, incubated in $0.05 \%$ trypsin (Gibco-Invitrogen, Carlsbad, CA, USA) in $0.1 \mathrm{mM}$ ethylenediaminetetraacetic acid $\left(20 \mathrm{~min}, 37^{\circ} \mathrm{C}\right)$; washed, cells were dissociated in phosphate-buffered saline (PBS) (x3) to eliminate cell debris and triturated in the same solution in a fire-polished Pasteur pipette. Cells that passed through a $100-\mu \mathrm{m}$ strainer (Falcon, Oxnard, CA, USA) were then seeded $\left(1 \times 10^{5} / \mathrm{ml}\right)$ into Falcon culture flasks and grown in medium containing 2\% B27 (Gibco-Invitrogen); $20 \mu \mathrm{g} / 1$ human recombinant basic fibroblast growth factor (bFGF) (Gibco-Invitrogen); $20 \mu \mathrm{g} / 1$ epidermal growth factor (EGF) (Gibco-Invitrogen); 2 mmol/1 L-glutamine; $4 \mathrm{U} / 1$ insulin and $100 \mathrm{U} / \mathrm{ml}$ penicillin/streptomycin (Gibco-Invitrogen), $\mathrm{pH}$ 7.2-7.5. The medium was changed every three days until primary tumor spheres were visible under microscopy within about 2 weeks. After primary tumor spheres reached the size of $\sim 100-200$ cells/sphere, the spheres were dissociated and plated in 96-well plates in $100 \mu \mathrm{l}$ volumes of serum-free neural stem cell medium. Every 2 days, $20 \mu 1$ neural stem cell medium was added to the culture. In these conditions, cells were able to grow in vitro in clusters called tumorspheres and maintained in an undifferentiated state, as indicated by morphology and expression of stem-cell markers such as CD133 and Nestin. Then, double immunofluorescence staining was performed to detect the expression of different markers.

Statistical analysis. The software of SPSS version 16.0 for Windows (SPSS Inc., Chicago, IL, USA) was used for statistical analysis. The Pearson's correlation was calculated between the expression levels of the CD133 or Nestin and blood vessels in glioblastoma tissues. $\mathrm{P}<0.05$ was considered to denote significant differences.

\section{Results}

Expression of CD133 and Nestin in glioblastoma tissues. In glioblastoma, we identified that CD133 or Nestin were not only expressed on GSCs but were also expressed in the brain capillaries as the endothelial cells (Fig. 1B-F and Fig. 2B-F, arrows). There were copious $\mathrm{CD}_{133^{+}}$or $\mathrm{Nestin}^{+}$blood vessels distributed in the region which $\mathrm{CD}_{133^{+}}$or Nestin ${ }^{+}$cells were accumulated (Fig. 1E, F and Fig. 2D, arrows). Some Nestin $^{+}$blood vessels even had a railway-like pattern with simultaneous labeling of the basal endothelial membrane and the outer border of the vessels (Fig. 2B and E, arrows). Accumulation of Nestin ${ }^{+}$blood vessels as a cluster was also observed (Fig. 2D, arrows). The percentage of $\mathrm{CD} 133^{+}$cells and $\mathrm{CD} 133^{+}$blood vessels were $24.75 \pm 6.71 \%$ and $18.01 \pm 5.77 \%$ in GBMs (Table I). The percentage of Nestin ${ }^{+}$cells and Nestin ${ }^{+}$ blood vessels were $26.67 \pm 7.94 \%$ and $14.08 \pm 5.09 \%$ (Table I). A positive correlation $(\mathrm{r}=0.482, \mathrm{P}=0.002)$ was found between the expressions of $\mathrm{CD} 133^{+}$cells and $\mathrm{CD} 133^{+}$blood vessels. Similarly, the expressions of Nestin ${ }^{+}$cells and Nestin ${ }^{+}$blood vessels were also positively correlated $(\mathrm{r}=0.390, \mathrm{P}=0.015)$.

Double immunofluorescence staining. The results of CD133/ CD31 and Nestin/CD31 double immunofluorescence staining, indicated that both the $\mathrm{CD}_{133^{+}}$cells and Nestin ${ }^{+}$cells accumulated around the $\mathrm{CD} 31^{+}$blood vessels. Moreover, some $\mathrm{CD} 133^{+}$ or Nestin ${ }^{+}$cells co-expressed CD31 (Figs. 3 and 4, arrows and inset). Double immunofluorescence for CD133/Nestin revealed that the $\mathrm{CD}_{133}{ }^{+} / \mathrm{Nestin}^{+}$GSCs were accumulated in the niches that had blood vessels distributed around the surroundings (Fig. 5, arrows and inset). In addition, we found that GSCs could co-express VEGF/CD31, CD133/CD31, CD133/Nestin and Nestin/CD31 (Figs. 6-9). These findings demonstrate that both $\mathrm{CD}_{133^{+}}$and Nestin ${ }^{+}$blood vessels could be detected which may originate from the differentiation of tumor stem cells. 


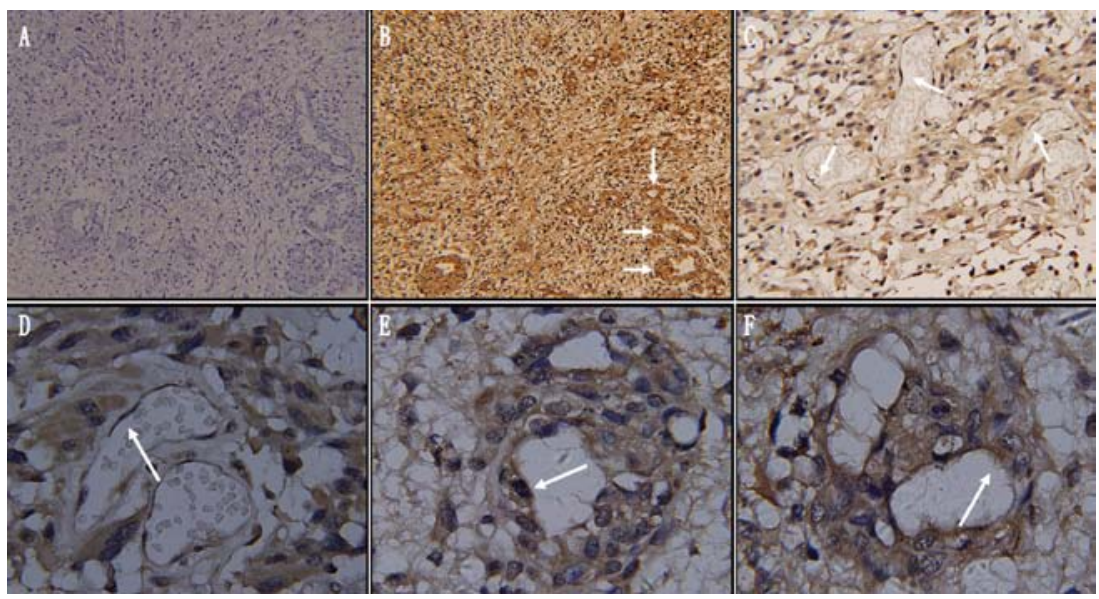

Figure 1. CD133 immunohistochemical staining in GBMs. (A) The specificity of the immunohistochemical labelling was controlled by primary antibody omission where no reactivity was observed. (B-F, arrows) CD133 was not only expressed on GSCs but also in the brain capillaries as the endothelial cells. (E-F, arrows) $\mathrm{CD}_{133^{+}}$cells accumulated around the blood vessels. (A) and (B), 10x objective; (C), 20x objective; (D) and (F), 100x objective.

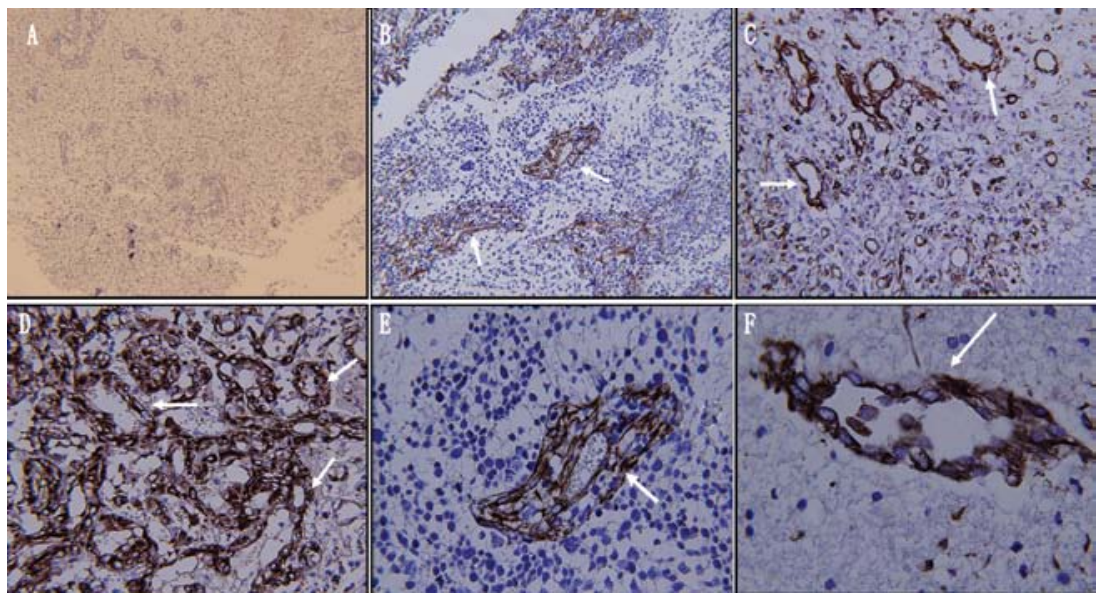

Figure 2. Nestin immunohistochemical staining in GBMs. (A) The specificity of the immunohistochemical labelling was controlled by primary antibody omission where no reactivity was observed. (B-F, arrows) Nestin was not only expressed on GSCs but also in the brain capillaries as the endothelial cells. (C) $\mathrm{Nestin}^{+}$cells accumulated around the blood vessels (arrows). Some Nestin ${ }^{+}$blood vessels even had a railway-like pattern with simultaneous labeling of the basal endothelial membrane and the outer border of $\mathrm{t}$ he vessels (B, E, arrows). (D) The Nestin ${ }^{+}$blood vessels could be accumulated as a cluster (arrows). (A), 10x objective; (B-D), 20x objective; (E) and (F), 100x objective.

Table I. Positive immunostaining of CD133 and Nestin in glioblastoma tissues.

\begin{tabular}{lccr}
\hline $\mathrm{CD}_{133}{ }^{+}$cells $(\%)$ & $\mathrm{CD} 133^{+}$blood vessels $(\%)$ & Nestin ${ }^{+}$cells $(\%)$ & Nestin $^{+}$blood vessels $(\%)$ \\
\hline $24.75 \pm 6.71^{\mathrm{a}}$ & $18.01 \pm 5.77$ & $26.67 \pm 7.94^{\mathrm{b}}$ & $14.08 \pm 5.09$
\end{tabular}

${ }^{\mathrm{a}} \mathrm{P}<0.05$ vs. $\mathrm{CD} 133^{+}$blood vessels $(\%) ;{ }^{\mathrm{b}} \mathrm{P}<0.05$ vs. Nestin ${ }^{+}$blood vessels $(\%)$. A total of 70 glioblastoma tissue specimens were evaluated.

\section{Discussion}

Cancer becomes clinically detectable only after a tumor mass undergoes continuous expansion. However, expansion of a tumor mass beyond the initial microscopic size of a non-angiogenic tumor is dependent on the recruitment of its own vascular supply, angiogenesis and blood vessel co-option $(11,12)$. When the tumor is newly formed (non-blood vessel time), pre-existing blood vessels could satisfy the nutrition which the tumor stem cells need. At the time of the infinite multiplication of GSCs, the microenvironment in which the GSCs are located is at a high anoxic condition (13). Based on this phenomenon, Diabira et al proposed the new concept of a neo-niche, which emphasizes the importance of hypoxia in driving tumor growth (14). Hypoxia results in the production of certain growth factors and cytokines that have been shown to induce angiogenesis in gliomas (15). For example, HIF-1 (hypoxia inducible factor-1) is one of the master regulators that 


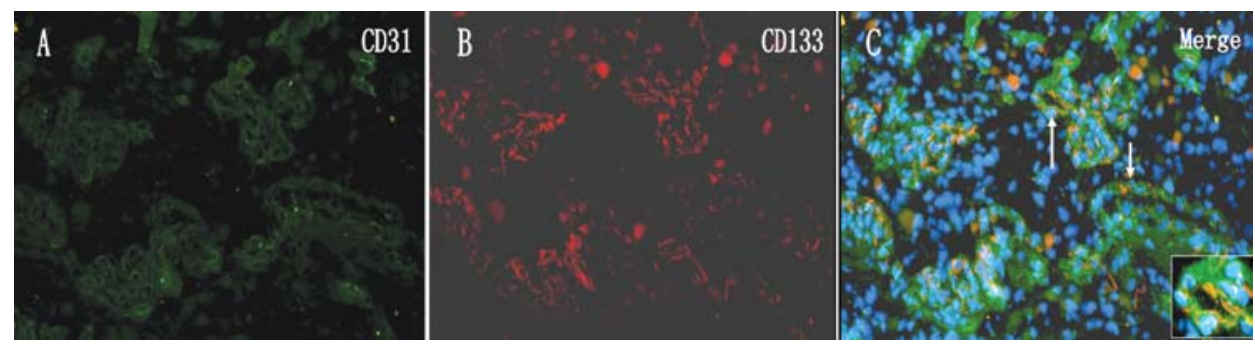

Figure 3. Double immunofluorescence staining of paraffin sections for CD31 (green) and CD133 (red) in GBMs. Nuclei are counterstained with DAPI (blue). The $\mathrm{CD} 133^{+}$cells accumulated around the CD31+ blood vessels. Some of the CD31+ blood vessels co-expressed CD133. (C, arrows and inset); (A-C), 40x objective; inset, 100x objective.

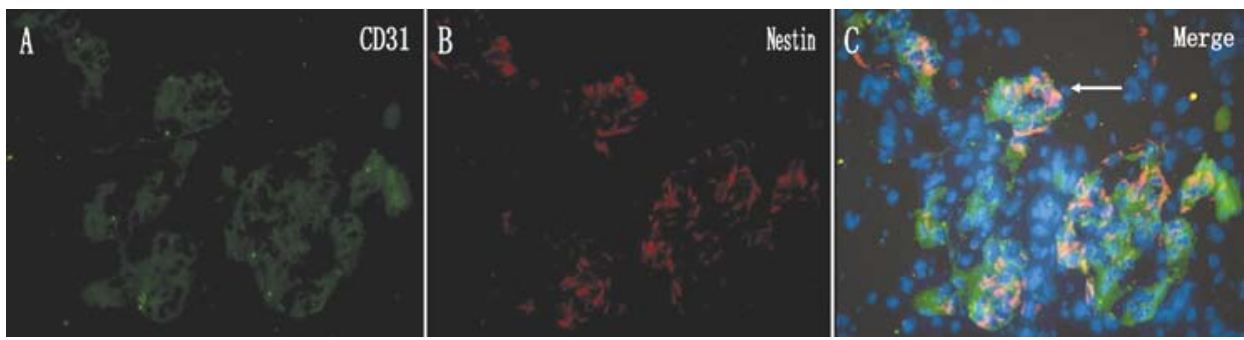

Figure 4. Double immunofluorescence staining of paraffin sections for CD31 (green) and Nestin (red) in GBMs. Nuclei are counterstained with DAPI (blue). Nestin $^{+}$cells accumulated around the CD31+ blood vessels. Some CD31+ blood vessels co-expressed Nestin. (C, arrow); (A-C), 40x objective.

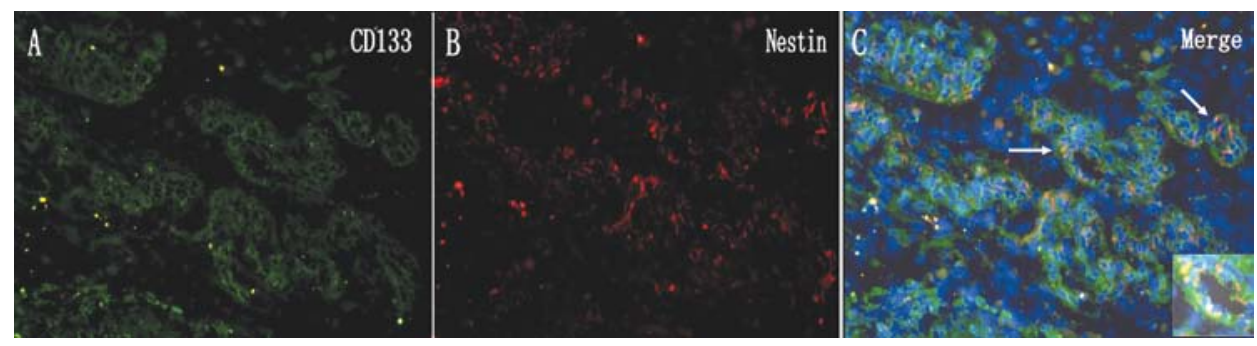

Figure 5. Double immunofluorescence staining of paraffin sections for CD133 (green) and Nestin (red) in GBMs. Nuclei are counterstained with DAPI (blue).

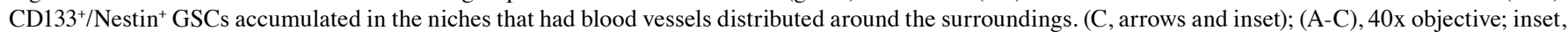
100x objective.

orchestrates the cellular response to hypoxia and has a central role in promoting proangiogenic and invasive properties in gliomas (16). Activation of HIF-1 results in the activation of VEGF and their receptors, platelet-derived growth factor-B (PDGF-B), matrix metalloproteinases, plasminogen activator inhibitor, transforming growth factors, angiopoietin, endothelin-1, inducible nitric oxide synthase, adrenomedullin and erythropoietin, all factors capable of promoting angiogenesis and invasion (17). Moreover, the CXCL12/CXCR4 biological axis may also be implicated in promoting angiogenesis, tumor cell proliferation and survival $(18,19)$. Therefore, these growth factors and cytokines have been shown to regulate both GSCs self-renewal and tumor vasculogenesis, further implying that these two processes are closely linked.

Cancer resembles a neo-organ, composed of various stages of developing cancer cells, including precancerous stem-like cells (pCSCs), cancer stem-like cells (CSCs) and cancer cells $(20,21)$. CSCs have been shown to promote tumor angiogenesis through secreting VEGF and via their potential for transdifferentiation into endothelial cells. In the present sudy, CD133 or Nestin staining of both tumor and endothelial cells confirms the recent results obtained by flow cytometry by Rebetz et al showing that $\mathrm{CD} 133^{+}$cells could have either a blood vessel or a glioma origin (22). These findings are in line with the recent description of two functional sub-groups of $\mathrm{CD}_{133^{+}}$cells in different types of cancer: one with a cancer-initiating potential as described in brain (23) and colon cancer (24) and one with an angiogenic potential as described in lung cancer (25). Furthermore, we have found the blood vessels distributed in the neighborhood of $\mathrm{CD} 133^{+}$or $\mathrm{Nestin}^{+}$niches and some CD31 ${ }^{+}$vessels co-expressed CD133 or Nestin (Figs. 3 and 4, arrows and inset). Together these findings suggest that both the $\mathrm{CD}_{133^{+}}$and Nestin ${ }^{+}$blood vessels found in the present study could represent newly formed vessels which may originate from the differentiation of the tumor stem cells in accordance with previous findings (26). Similarly, Wang et al (27) found that a subpopulation of cells within the glioma can give rise to endothelial cells 


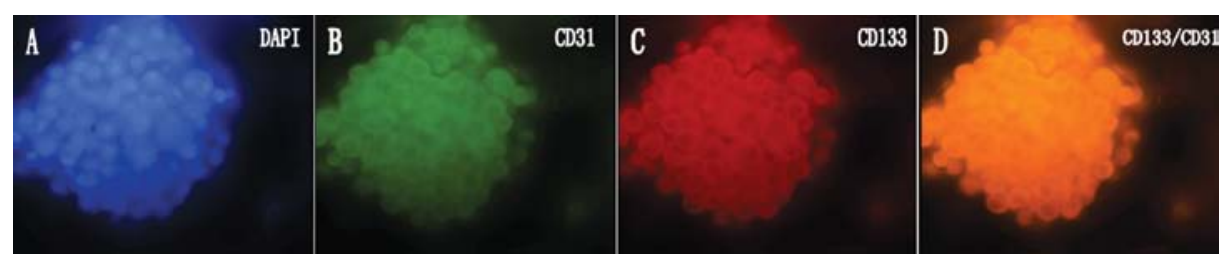

Figure 6. Double immunofluorescence staining of GSCs for CD31 (green) and CD133 (red). Nuclei are counterstained with DAPI (blue). The GSCs coexpressed both CD133 and CD31. (A-D), 40x objective.

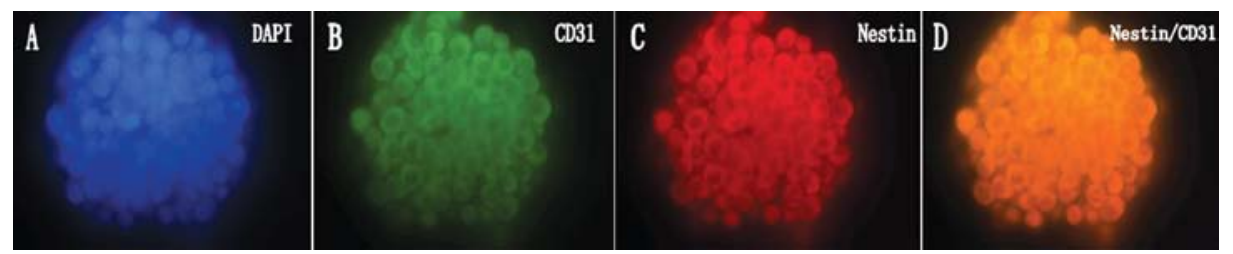

Figure 7. Double immunofluorescence staining of GSCs for CD31 (green) and Nestin (red). Nuclei are counterstained with DAPI (blue). The GSCs coexpressed both Nestin and CD31. (A-D), 40x objective.

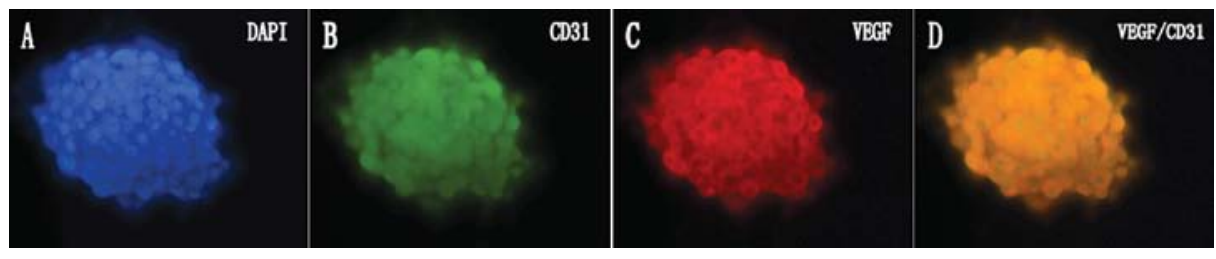

Figure 8. Double immunofluorescence staining of GSCs for CD31 (green) and VEGF (red). Nuclei are counterstained with DAPI (blue). The GSCs coexpressed both VEGF and CD31. (A-D), 40x objective.

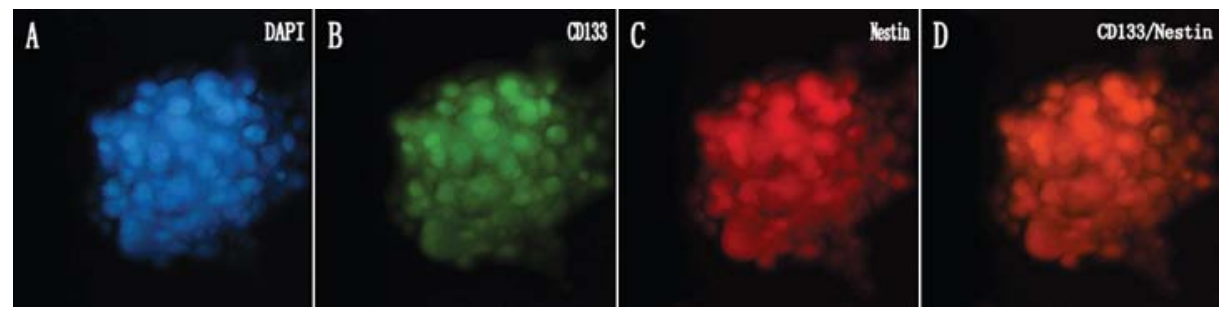

Figure 9. Double immunofluorescence staining of GSCs for CD133 (green) and Nestin (red). Nuclei are counterstained with DAPI (blue). The GSCs coexpressed both Nestin and CD133. (A-D), 40x objective.

via a bipotential progenitor intermediate, and that the $\mathrm{CD} 133^{+}$ cancer stem-cell-like fraction includes a population of endothelial progenitors. Hence, the endothelial differentiation of GSCs may be the reason why the $\mathrm{CD} 133^{+}$or Nestin ${ }^{+}$cells were found to not only be distributed around but also inside the blood vessels. In addition, we found a positive correlation between the expressions of $\mathrm{CD} 133^{+}$GSCs and CD133+ blood vessels $(\mathrm{P}=0.002)$ and between the expressions of Nestin ${ }^{+}$ GSCs and Nestin ${ }^{+}$blood vessels $(\mathrm{P}=0.015)$.

Ricci-Vitiani et al (26) have indicated that GSC-derived angiogenesis is essential for tumor survival. The microvascular system not only supplies the nutrition required for GSCs growth, but also improves its ability of self-renewal and proliferating. Thus, the microvascular system may protect GSCs from chemotherapies and radiotherapies, enabling these cells to reform a tumor mass following an initial clinical response. For example, solid tumor xenografts grown in mice with radiation-resistant endothelial cells are much less sensitive to radiation damage than tumors grown in wild-type mice (28). Moreover, endothelial cells were also found to be related to the recrudescence and metastasis of the glioma.

In summary, our data demonstrate that GSCs play a key role in contributing to the tumor vascularization by endothelial cell differentiation. This finding aids in the understanding of tumor vascularization and has important implications in the treatment strategy. An in-depth understanding of the lineage relationship between tumor stem-like cells and endothelial progeny should provide new insights into CSC biology and offer new therapeutic options for the anti-vascular treatment strategy. 


\section{Acknowledgements}

This study was supported by grant no.30672166 from the National Natural Science Foundation of China (NSFC). We would like to thank all surgeons from the Neurosurgery Department of Anhui Provincial Hospital for helping us collect tumor samples.

\section{References}

1. Stupp R, Mason WP, van den Bent MJ, et al: Radiotherapy plus concomitant and adjuvant temozolomide for glioblastoma. $\mathrm{N}$ Engl J Med 352: 987-996, 2005.

2. Singh SK, Clarke ID, Terasaki M, et al: Identification of a cancer stem cell in human brain tumors. Cancer Res 63: 5821-5828, 2003.

3. Calabrese C, Poppleton H, Kocak M, et al: A perivascular niche for brain tumor stem cells. Cancer Cell 11: 69-82, 2007.

4. Bao S, Wu Q, Sathornsumetee S, et al: Stem cell-like glioma cells promote tumor angiogenesis through vascular endothelial growth factor. Cancer Res 66: 7843-7848, 2006.

5. Folkman J: Angiogenesis in cancer, vascular, rheumatoid and other disease. Nat Med 1: 27-31, 1995.

6. Bergers $\mathrm{G}$ and Benjamin LE: Tumorigenesis and the angiogenic switch. Nat Rev Cancer 3: 401-410, 2003.

7. Kerbel RS: Tumor angiogenesis: past, present and the near future. Carcinogenesis 21: 505-515, 2000.

8. Pezzolo A, Parodi F, Corrias MV, et al: Tumor origin of endothelial cells in human neuroblastoma. J Clin Oncol 25: 376-383, 2007.

9. Dome B, Hendrix MJ, Paku S, et al: Alternative vascularization mechanisms in cancer: pathology and therapeutic implications. Am J Pathol 170: 1-15, 2007.

10. Bruno S, Bussolati $\mathrm{B}$, Grange $\mathrm{C}$, et al: $\mathrm{CD} 133^{+}$renal progenitor cells contribute to tumor angiogenesis. Am J Pathol 169: 2223-2235, 2006.

11. Pezzella F, Pastorino U, Tagliabue E, et al: Non-small cell lung carcinoma tumor growth without morphological evidence of neo-angiogenesis. Am J Pathol 151: 1417-1423, 1997.

12. Wesseling P, Ruiter DJ and Burger PC: Angiogenesis in brain tumors; pathobiological and clinical aspects. J Neurooncol 32: 253-265, 1997.

13. Salmaggi A, Boiardi A, Gelati M, et al: Glioblastoma-derived tumorospheres identify a population of tumor stem-like cells with angiogenic potential and enhanced multidrug resistance phenotype. Glia 54: 850-860, 2006.
14. Diabira S and Morandi X: Gliomagenesis and neural stem cells: key role of hypoxia and concept of tumor 'neo-niche'. Med Hypotheses 70: 96-104, 2008.

15. Tuettenberg J, Friedel C and Vajkoczy P: Angiogenesis in malignant glioma - a target for antitumor therapy? Crit Rev Oncol Hematol 59: 181-193, 2006.

16. Fujiwara S, Nakagawa K, Harada $\mathrm{H}$, et al: Silencing hypoxia inducible factor- $1 \alpha$ inhibits cell migration and invasion under hypoxic environment in malignant gliomas. Int J Oncol 30: 793-802, 2007.

17. Kaur B, Khwaja FW, Severson EA, et al: Hypoxia and the hypoxia-inducible-factor pathway in glioma growth and angiogenesis. Neuro Oncol 7: 134-153, 2005.

18. Pan J, Mestas J, Burdick MD, et al: Stromal derived factor-1 (SDF-1/CXCL12) and CXCR4 in renal cell carcinoma metastasis. Mol Cancer 5: 56, 2006

19. Bachelder RE, Wendt MA and Mercurio AM: Vascular endothelial growth factor promotes breast carcinoma invasion in an autocrine manner by regulating the chemokine receptor CXCR4. Cancer Res 24: 7203-7206, 2002.

20. Chen L, Shen R, Ye Y, et al: Precancerous stem cells have the potential for both benign and malignant differentiation. PLoS One 2: e293, 2007.

21. Hong D, Gupta R, Ancliff P, et al: Initiating and cancerpropagating cells in TEL-AML1-associated childhood leukemia. Science 319: 336-339, 2008

22. Rebetz J, Tian D, Persson A, et al: Glial progenitor-like phenotype in low-grade glioma and enhanced CD133-expression and neuronal lineage differentiation potential in high-grade glioma. PLoS One 3: e1936, 2008.

23. Singh SK, Hawkins C, Clarke ID, et al: Identification of human brain tumour initiating cells. Nature 432: 396-401, 2004.

24. Ricci-Vitiani L, Lombardi DG, Pilozzi E, et al: Identification and expansion of human colon-cancer-initiating cells. Nature 445: 111-115, 2007.

25. Hilbe W, Dirnhofer S, Oberwasserlechner F, et al: CD133 positive endothelial progenitor cells contribute to the tumour vasculature in non-small cell lung cancer. J Clin Pathol 57: 965-969, 2004

26. Ricci-Vitiani L, Pallini R, Biffoni M, et al: Tumor vascularization via endothelial differentiation of glioblastoma stem-like cells. Nature 468: 824-828, 2010.

27. Wang R, Chadalavada K, Wilshire J, et al: Glioblastoma stemlike cells give rise to tumor endothelium. Nature 468: 829-833, 2010.

28. Garcia-Barros M, Paris F, Cordon-Cardo C, et al: Tumor response to radiotherapy regulated by endothelial cell apoptosis. Science 300: 1155-1159, 2003. 